\title{
Gradhiva
}

GRADHIV

Revue d'anthropologie et d'histoire des arts

$15 \mid 2012$

Robots étrangement humains

\section{Androïde cherche humain pour contact électrique}

Les cinétiques de l'attachement en robotique

Android in search of a human for electrical contact. The kinetics of binding in robotics

\section{Emmanuel Grimaud}

\section{OpenEdition}

\section{Journals}

Édition électronique

URL : http://journals.openedition.org/gradhiva/2328

DOI : $10.4000 /$ gradhiva. 2328

ISSN : $1760-849 x$

\section{Éditeur}

Musée du quai Branly Jacques Chirac

\section{Édition imprimée}

Date de publication : 16 mai 2012

Pagination : 76-101

ISBN : 978-2-35744-047-0

ISSN : 0764-8928

\section{Référence électronique}

Emmanuel Grimaud, « Androïde cherche humain pour contact électrique », Gradhiva [En ligne],

15 | 2012, mis en ligne le 16 mai 2015, consulté le 19 avril 2019. URL : http://journals.openedition.org/ gradhiva/2328; DOl : 10.4000/gradhiva.2328 


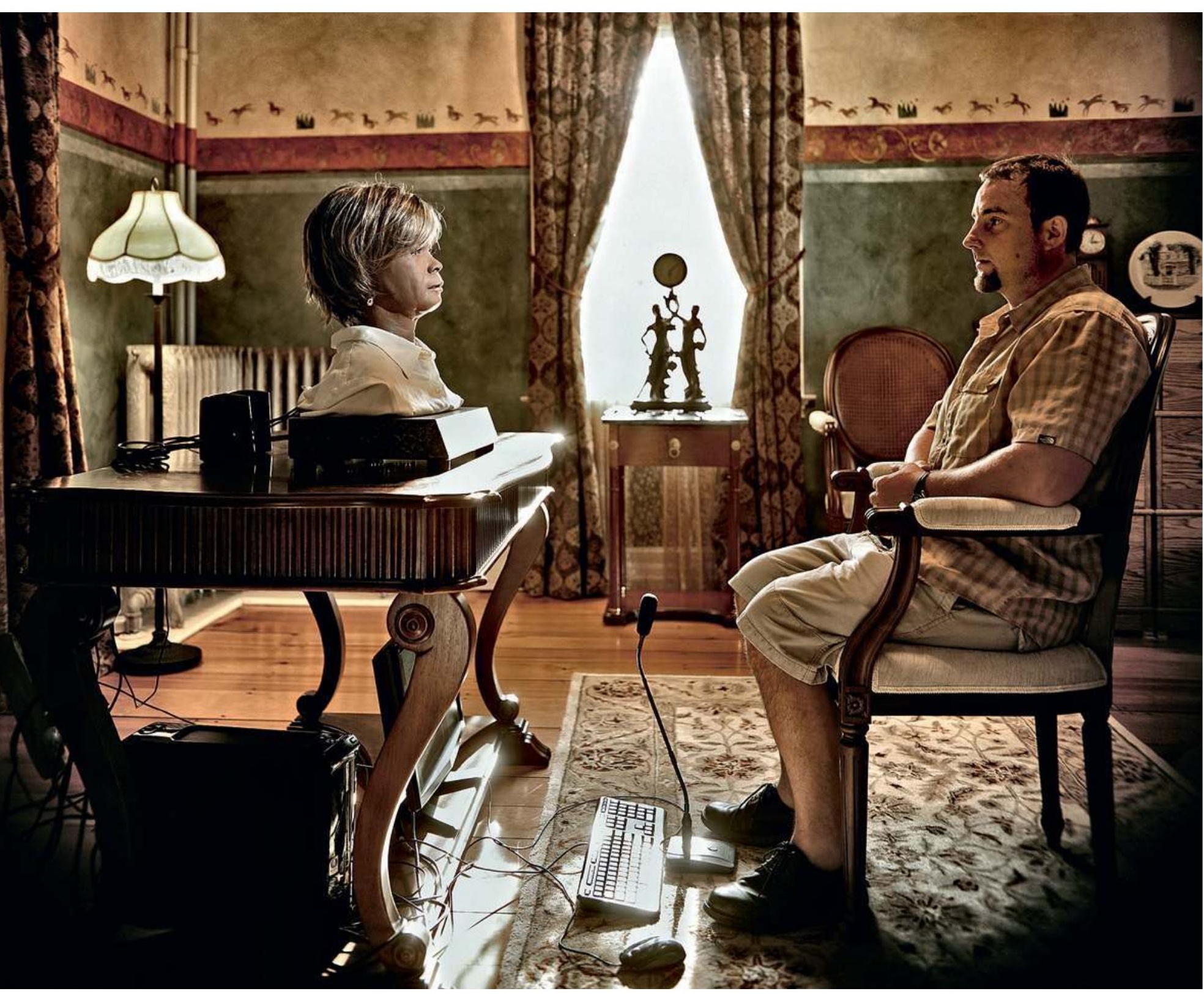

Fig. 1 Nick Mayer, membre du projet LifeNaut dans le Vermont, assis face à la tête-robot Bina48. Hanson Robotics a créé cet humanoïde parlant à l'image de Bina Rothblatt, co-fondatrice de LifeNaut @ Max Aguilera-Hellweg. 


\section{Androïde cherche humain contact électrique Les cinétiques de l'attachement en robotique}

Emmanuel Grimaud

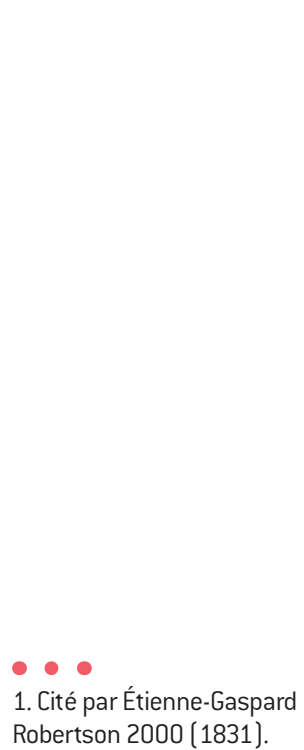

Alors s'élève, du plancher même, une figure blanche qui grandit par degré jusqu'à la proportion humaine... le fantôme devient de plus en plus visible, resplendissant... Il se promène, il s'approche, il se penche vers vous : vous frémissez, il s'avance encore, vous allez le toucher, il disparait et vous vous retrouvez dans les mêmes ténèbres.

Un visiteur de fantasmagorie, Paris, $1793^{1}$
Introduction

La robotique humanoïde nous convie à un bien étrange exercice qui consiste à faire «table rase » de l'être humain. S'agit-il d'une manière de cligner des yeux? de bouger la tête? un mode de locomotion? des capacités cognitives et, si c'est le cas, lesquelles? Ces questions, les roboticiens ne cessent de se les poser lorsqu'ils conçoivent leurs créatures. Et elles se reposent nécessairement de manière aiguë toutes les fois qu'on entre en relation avec un humanoïde. Avec une créature anthropomorphique, bien plus peut-être qu'avec une autre, s'instaure une parade d'approche quasi animale, marquée par des temps d'observation, d'attente, des phases d'attraction et de répulsion. Il faut se faire son diagnostic sur l'être artificiel auquel on a affaire, évaluer ses possibilités, ses limites et sa manière à lui d'être présent. Le jeu qui consiste à cultiver la confusion des genres (entre l'humain et la machine) et à voir combien de temps on peut l'entretenir admet de multiples versions scientifiques (le test de Turing étant le plus connu) autant que de versions populaires, les robots faisant l'objet d'innombrables démonstrations de foire. On peut même aller plus loin. Ce jeu métaphysique est sans doute l'un 


\section{- $\bullet$}

2. On pourrait citer bien d'autres exemples, de l'automate joueur d'échecs de Johann Wolfgang von Kempelen (fin du xvIII siècle), dont le mystère exceptionnellement orchestré par son concepteur reposa sur l'incertitude quant à la présence ou non d'un trucage (Schaffer 1999), aux karakuri japonais, ces petits artefacts mécaniques populaires au $\mathrm{xvII}^{\mathrm{e}}$ siècle (dont le serveur de thé et l'archer, les figures les plus connues, sont discutées par Zaven Paré dans ce même dossier], mis en marche par leurs propriétaires devant leurs invités pour tester leur perspicacité à deviner le mécanisme de leur prouesse.

3. L'exemple se trouve aussi discuté par Paul Veyne dans Les Grecs ont-ils cru à leurs mythes? Paris, Seuil, 1992. des plus excitants jamais conçus par l'humanité qui se regarde ainsi dans un miroir déformant, à tel point qu'on peut se demander si ce n'est pas au fond pour susciter ce genre d'émotion qu'on les conçoit.

Dans un article intitulé "Comment se fixe la croyance?» (1878), Charles Sanders Peirce s'interrogea sur cette tendance très humaine à vouloir à tout prix détruire le doute quand celui-ci s'installe, alors que remettre en cause des croyances bien installées est un sport beaucoup moins naturel. Or, dans l'histoire de la mécanique et des automates, les exemples ne manquent pas où la confusion des genres (humain/machine) est la motivation même qui pousse à leur fabrication. Elle s'inscrit au cœur de l'artefact, elle se cultive, on fait tout pour que le doute s'installe et pour le faire durer. Le fameux test imaginé par Alan Turing, largement glosé par ceux qui s'intéressent à l'histoire des machines pensantes (Gunderson 1964; Anderson 1983), ne fut inspiré par rien d'autre qu'un jeu de foire dont l'objectif était de générer le maximum d'ambiguïté pour tromper le questionneur. Il visait à tester les capacités d'une machine à nous confondre non seulement sur son identité de machine, mais aussi sur sa masculinité ou sa féminité. Dans la conception de créatures artificielles, la confusion ontologique est motrice ${ }^{2}$. Il faut soit la cultiver, soit la dépasser.

La plupart des artefacts robotiques tirent parti d'un mécanisme de croyance familier des anthropologues et que le psychanalyste Octave Mannoni avait résumé dans une phrase éloquente : "Je sais bien, mais quand même... " (1969) Je sais bien, le père Noël n'existe pas, mais quand même', j'y crois. Je sais bien, ce n'est qu'une pierre, mais quand même, cela peut être à certains moments, dans certaines conditions, une divinité. Je sais bien, ce n'est qu'une machine, mais quand même elle bouge, elle parle, elle me répond. Cette maxime s'applique sans difficulté aux robots les plus contemporains, et plus largement à un grand nombre d'artefacts technologiques. Je sais bien, mon ordinateur n'est qu'un assemblage de programmes, mais quand même c'est aussi mon compagnon. Beaucoup des attachements noués par les humains avec des choses, avec des dieux comme avec des interfaces technologiques, dans les cultures les plus variées, se développent ainsi, sur le mode du "comme si ", autorisant l'ambiguïté, le flou ou une certaine flexibilité qui permet de ne pas trancher une fois pour toutes sur leur identité. Comment l'univers du "comme si", avec tout ce que cela suppose de possibilités d'incarnations hypothétiques, s'accommode-t-il de la tendance à vouloir dissiper les doutes mise à jour par Peirce? De la même façon que c'est l'usage qu'on fait des masques katchinas (pour reprendre l'exemple de Mannoni) qui les fait sortir de leur sommeil, les robots sont dépendants des jeux d'attribution auxquels leurs utilisateurs les soumettent. On ne peut se contenter de prendre acte de cette dépendance, il faut examiner de plus près, à partir de cas concrets, la spécificité de l'interaction dans laquelle un humanoïde nous embraie. À la différence d'une peinture ou d'une sculpture, qui invitent à une relation contemplative, un robot incite d'emblée à l'action ou à l'interaction sur le mode du jeu. Le problème de l'attribution d'un statut, d'une identité, voire d'une âme, à un humanoïde ne peut être abordé sérieusement qu'en observant de près, en temps réel, la manière dont les attachements se font et se défont parfois aussi vite.

Que doit-on entendre ici par attachement? Il s'agit d'un terme suffisamment vague pour permettre tous les degrés d'intensité, englobant aussi bien les relations les plus utilitaires aux objets, l'amitié ou l'amour de son prochain que les dépendances plus ambiguës ou difficiles à qualifier comme celles que l'on peut entretenir avec un ordinateur ou un animal de compagnie. À en croire la «robo- 
psychologie" (Libin 2004), si on s'attache à des machines, c'est parce qu'on les confond toujours à un moment ou un autre avec d'autres êtres vivants (des humains, des animaux). L'attribution de caractéristiques anthropomorphiques à autre chose qu'à des humains est assumée ici comme une tendance profondément ancrée dans le genre humain. Ce dernier se modèle sur ce qu'il connaît déjà pour entrer en relation avec les objets qui lui sont offerts. La robotique n'aurait plus qu'à jouer sur ces processus d'attribution et faire de "l'anthropomorphisme appliqué" (Braitenberg 1984; Heider et Simmel1944). Mais a-t-elle une vision claire de ces processus d'attribution souvent complexes? Et que faut-il pour que l'attribution se mue en attachement, voire en rapport passionnel?

À première vue, il n'est pas besoin de faire des expériences en laboratoire pour cela, on en fait tous plus ou moins l'expérience au quotidien avec les ordi-

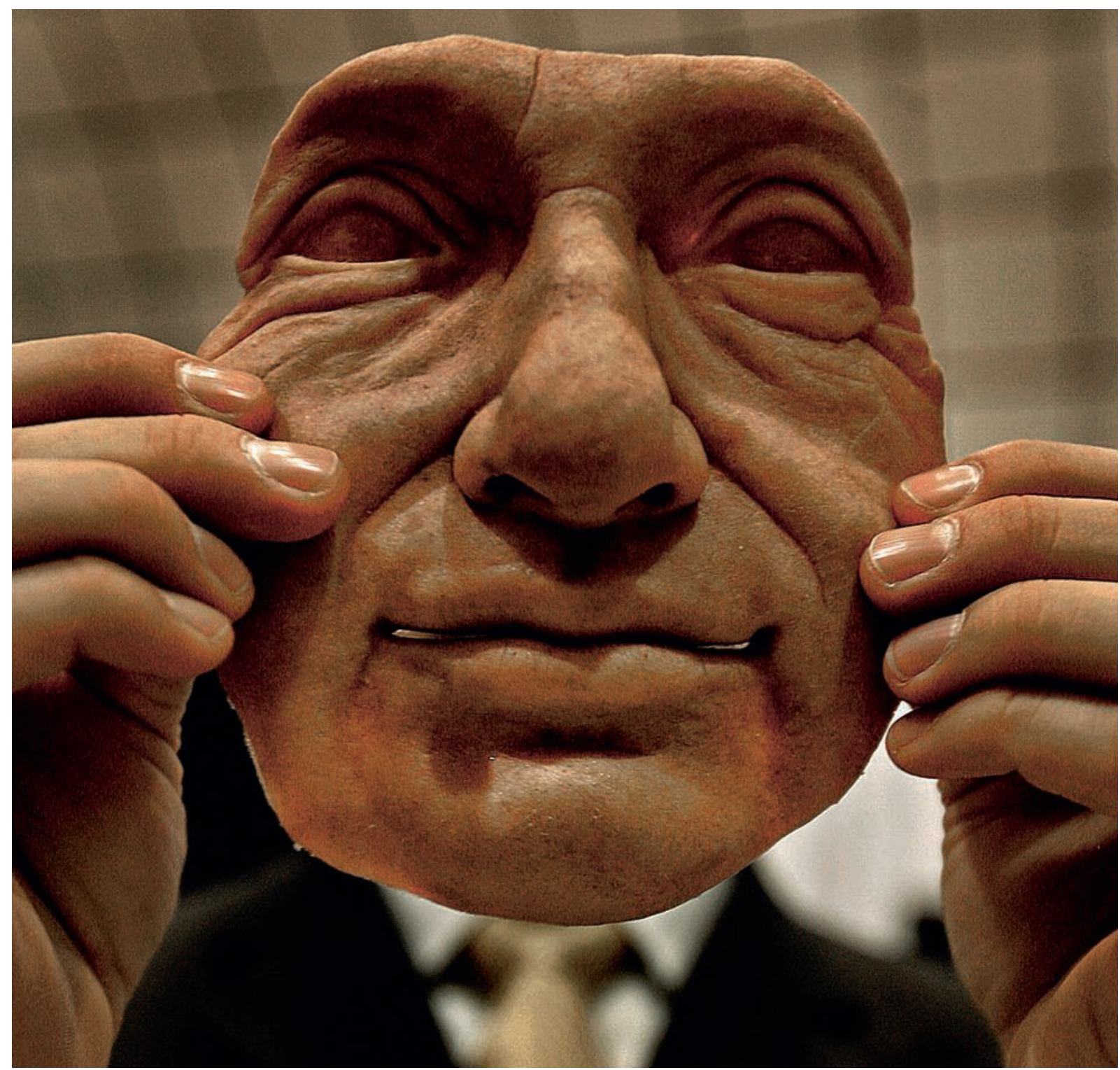

Fig. 2 Matthew W. Fisher avec les chercheurs de Hanson Robotics tient un visage artificiel, Boston, 2007 @ Photo David L. Ryan/Boston Globe via Getty Images. 
nateurs ou d'autres objets qui nous entourent et dont nous nous sentons dépendants. Plus l'objet est programmable et gagne en répondant (ou en interactivité), plus on multiplie les possibilités d'attribution et le moyen de créer de l'accoutumance. De nombreux travaux, dont il serait difficile de faire l'inventaire tant il en existe, lient les technologies et les passions bien avant le développement de la robopsychologie comme un champ à part entière. De nombreuses études comparent chez les enfants autant que chez les adultes l'attachement aux objets, aux animaux de compagnie, l'amour des machines et des ordinateurs. On y voit les rapports utilitaires aux objets qui nous entourent se muer en relations passionnelles, la technologie coloniser l'intimité et toute relation à un objet technologique tendre vers la dépendance ou l'accoutumance, et cela bien au-delà de l'univers des enfants ou des communautés de hackers qui furent les premiers concernés par ce genre d'étude (Turkle 2005). Jamais on n'a autant décortiqué cette multitude d'attachements que depuis que la robotique en a fait l'une de ses préoccupations principales. Mais qu'y a-t-il dans l'électronique qui soit si propice à la passion? Est-ce simplement le surplus d'animation qu'elle offre, la petite vibration qui rend les objets de notre entourage plus vivants?

\section{À propos d'un robot qui ne pouvait être humain que par accident}

On est parfois déçu par les performances des humanoïdes, sans doute parce qu'on en attend beaucoup, surtout lorsqu'ils nous ressemblent. Cependant, on peut être surpris par les mécanismes psychologiques d'une grande plasticité par lesquels on en vient à s'attacher à eux, à leur attribuer une place, un rôle, des vertus, même lorsque leurs programmes déraillent et nous renvoient à leur consistance de machines. Un robot rétrograde aussi vite qu'on l'a surclassé dès lors qu'il se casse ou ne fonctionne plus. Dans «Saya ou l'impossible virtuosité " (2011), Zaven Paré raconte une démonstration d'un roboticien qui dérape. Le robot est une institutrice japonaise qui interagit avec les enfants d'une salle de classe. Il faut lire la très belle description de l'auteur jusqu'au moment où le robot répète le même discours et les mêmes questions en boucle. La séance tourne alors au ridicule. Le roboticien ne sait plus quoi faire, il n'est ni metteur en scène ni prestidigitateur. Les enfants se jettent sur la pauvre institutrice et la déshabillent, dévoilant ses câbles et sa peau en latex. La confusion ontologique est rompue, la supercherie, dévoilée. L'institutrice n'était qu'un pauvre programme fait pour mener les enfants en bateau. La jolie mise en scène de l'androïde venue civiliser les enfants bascule dans un théâtre de la cruauté. Il y a toujours un moment où le robot nous ramène à sa nature de machine, il n'y a rien à faire. Il casse, dérape, sort de l'interaction à laquelle il a été préparé. "Après tout, ce n'est qu'une machine! » se dit-on alors. L'effet d'humanité d'un humanoïde ne s'attribue pas une fois pour toutes - leurs concepteurs le savent bien-, il se conquiert et il s'agit de savoir le conserver. Un téléviseur qui émet parvient à se faire oublier au point qu'on discute des informations ou du contenu d'un film sans se soucier de l'émetteur. Mais, dès lors qu'il n'émet plus, il n'est plus qu'un amas de circuits et un tube cathodique pour l'utilisateur, de la même façon qu'un ordinateur rétrograde et devient profondément étranger quand il ne fonctionne plus. Entre l'objet utilitaire doté de toutes ses capacités et l'objet défaillant, l'écart semble moins important qu'entre l'humanoïde assimilé, même un court instant, à une personne en chair et en os et l'humanoïde détraqué qui reste embourbé dans sa condition de 
Fig. 4 Mécanisme de l'automate et tympanon par Kintzing et Roentgen, 1784 (c) Musée des arts et métiers, Paris/photo F. Delastre. non verbale dans l'interaction humaine. Mais pour les industriels qui le finançaient et pour le grand public, le Geminoid était la matérialisation la plus aboutie d'un fantasme de dédoublement, la concrétisation physique la plus proche, dans la robotique actuelle, de l'idée du clone. Dans un avenir proche, nous pourrions tous avoir notre Geminoid pour nous remplacer dans une conférence lorsque nous sommes absents, disait son concepteur. On peut s'interroger sur la véritable innovation technique d'Ishiguro, et nous l'avons fait dans un livre détaillé sur le sujet (Grimaud et Paré 2011). Le Geminoid était le produit d'un petit glissement, mais qui est peut-être un grand écart, comparable comme on le verra par la suite à celui qui consiste à passer pour les enfants d'une peluche à un animal artificiel et, pour les adultes, d'un sex toy à un mannequin robotisé. Le roboticien multiplia les expériences autour de son système de téléopération à visage humain pour démontrer tout le gain que l'on peut espérer d'une interface anthropomorphique, y compris dans d'autres contextes que son laboratoire. Pour travailler à améliorer sa créature, il fit venir de nombreuses personnes issues des sciences cognitives et de la psychologie expérimentale, mais aussi des artistes. Il plaça son humanoïde pendant plusieurs mois dans la cafétéria d'une grande foire d'art contemporain et n'hésita pas à jouer le jeu d'interviews spectaculaires, répondant aux questions d'un journaliste par l'intermédiaire de son robot avant de le faire s'éteindre de manière dramatique. «Maintenant, laissez-moi dormir! " disait-il pour clore l'interview, avant de basculer son corps en arrière et d'ouvrir grand la bouche comme s'il agonisait. Le Geminoid devint très vite une star de la robotique humanoïde japonaise, mais il n'était au fond rien de plus qu'une grosse marionnette avec des capteurs. Son concepteur donna un jour une conférence où il reconnut que son robot ne pouvait être assimilé à autre chose qu'à un handicapé cérébral, vu l'état actuel de nos capacités à reproduire le cerveau humain. Mais Ishiguro ne prétendait pas travailler sur ce terrain. Son objectif scientifique était ailleurs. Il s'agissait d'une part de reproduire l'apparence humaine avec un sens accru du détail et du micro-mouvement, et d'étudier le rôle de ces micro-expressions dans l'interaction humaine. Ishiguro, qui connaissait bien le travail de Masahiro Mori sur l'uncanny valley, s'interrogeait sur le rôle joué par la " confusion ontologique " dans l'interaction avec une machine. Si cette confusion ne dure que quelques secondes, durant le premier contact avec le robot, que faire lorsque la supercherie est démasquée? Faut-il tout faire pour maintenir la confusion? Ou peut-on continuer à interagir avec lui, et sur quel mode? Le Geminoid était typiquement, comme beaucoup d'objets robotiques, un objet-frontière, titillant les limites de la communication humaine. Il aurait bien pu finir comme Saya, démantelé dans une salle de classe, si Ishiguro n'était pas aussi un grand metteur en scène, capable de faire mourir son robot devant un public pour lui donner un petit choc métaphysique. C'est que le Geminoid avait une vertu remarquable d'un point de vue anthropologique. Il obligeait ceux qui interagissaient avec lui à se concentrer sur des choses auxquelles on ne fait plus attention, dans l'interaction ordinaire entre deux êtres humains, qu'à de rares occasions : un battement de cils, un changement de direction dans le regard, un frémissement des lèvres. Le plus petit changement dans la plasticité du visage du Geminoid était un événement.

C'est sans doute par accident que le Geminoid a révélé ses leçons les plus instructives. Alors que les expérimentateurs tentaient de communiquer par son intermédiaire, le robot passait son temps à dérailler, ouvrait la bouche comme un poisson, se bloquait et transmettait la voix de son contrôleur avec un temps de retard. Même dans ce contexte, les expérimentateurs trouvèrent le moyen 


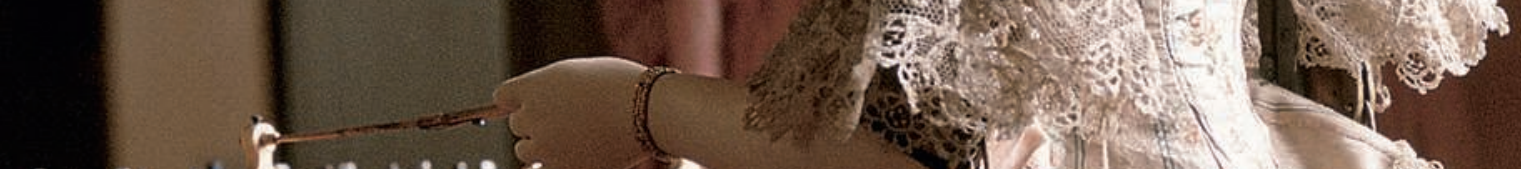

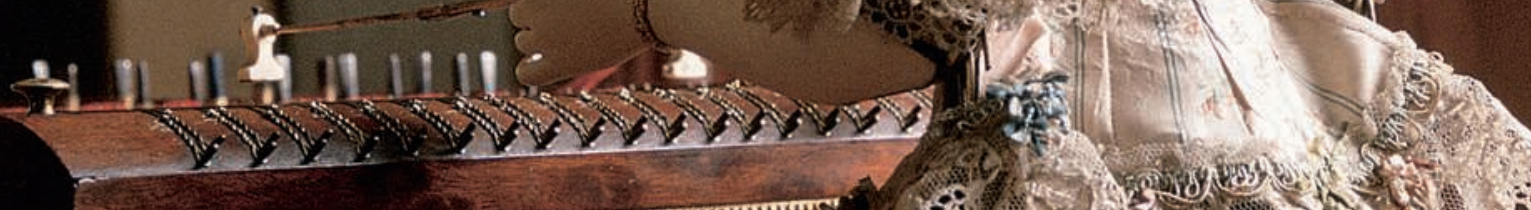




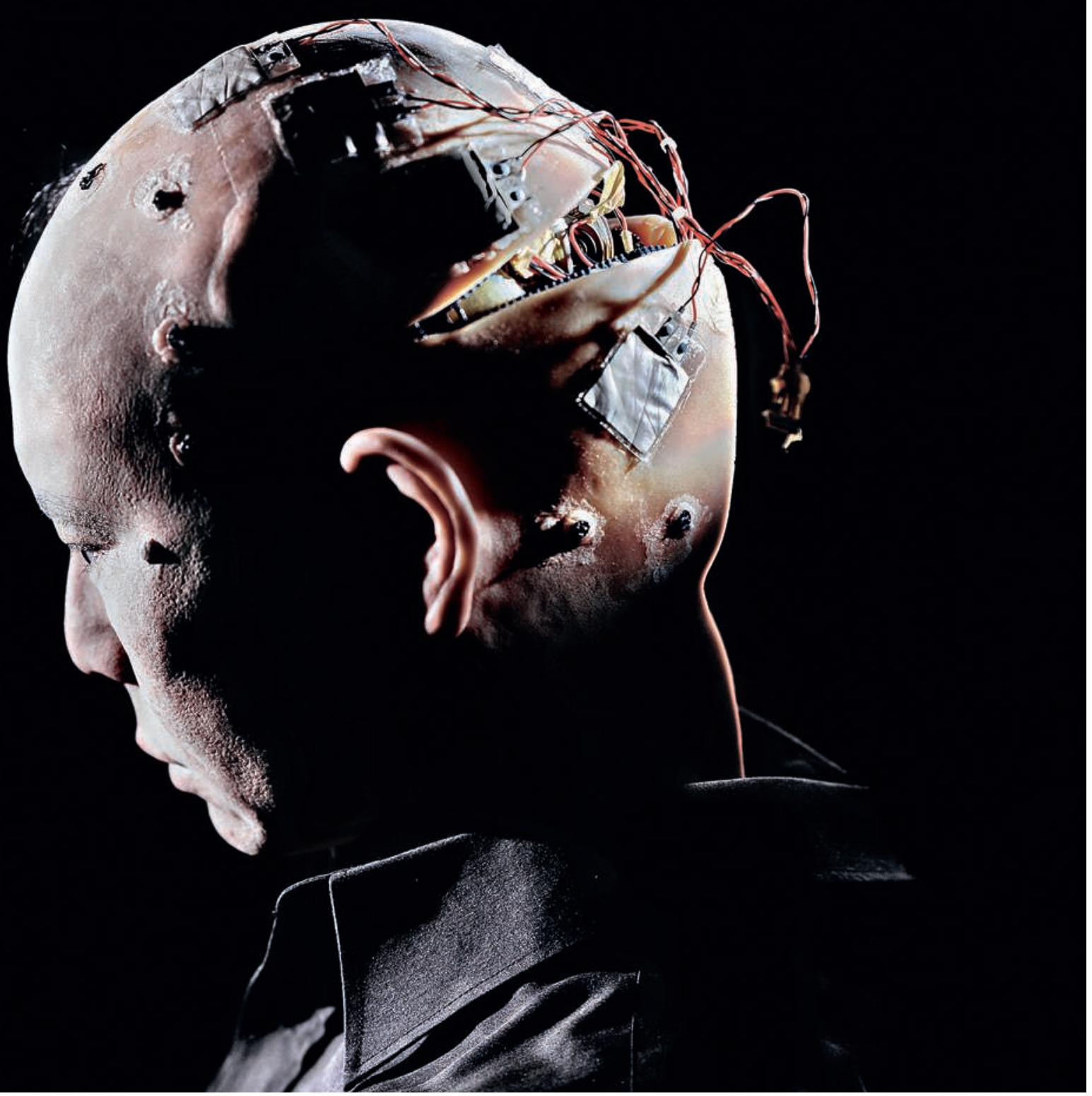

Fig. 5 Le roboticien Hiroshi Ishiguro de l'université d'Osaka a fabriqué son propre jumeau mécanique () Max Aguilera-Hellweg.

de continuer leur communication par l'intermédiaire de cette créature aussi confuse que maladroite et de se prêter au jeu d'une longue interaction en assumant que le Geminoid n'était qu'une machine. Plus le robot traduisait mal les intentions de son contrôleur, plus il gagnait en autonomie, plus les interlocuteurs en jouaient et plus la conversation ouvrait de possibilités d'identification. C'est que la confusion ontologique et son corollaire, la rétrogradation, qui semblait ailleurs irréversible (et notamment dans le cas de Saya), opéraient en fait de manière cyclique dans l'interaction. En effet, si le moment où l'on se demandait s'il s'agissait d'un humain ou d'une machine ne durait que quelques secondes, la confusion ne cessait de se réinstaller de façon nouvelle au cours du dialogue, au détour d'un petit geste du robot ou d'un clignement de l'œil qui paraissait particulièrement réaliste à son interlocuteur. L'interaction avec un tel robot était en fait rythmée par une boucle marquée par une confusion initiale (humain ou robot? se demandait-on au premier contact), suivie d'une séparation (où l'interlocuteur faisait bien la différence entre l'humain et la machine), puis d'une phase beaucoup plus aléatoire marquée par des confusions accidentelles. Dans cette dernière phase, de petits gestes, des postures, des regards pouvaient être inter- 


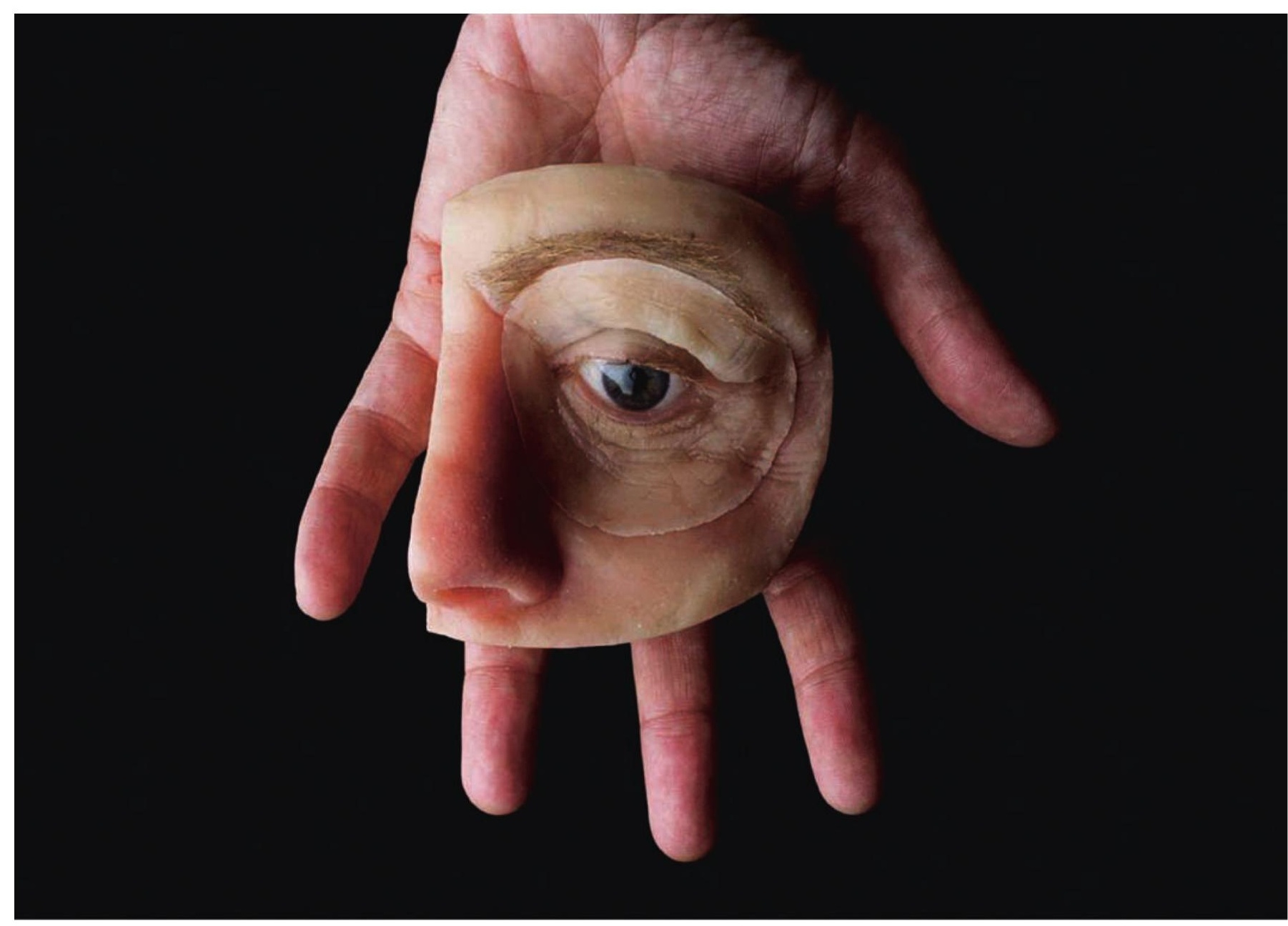

Fig. 6 Robot utilisant des pièces magnétiques fabriqué par la Virchow Campus Klinik, université Humboldt, Berlin, 2007 @ Peter Menzel/Cosmos.

prétés comme étant humains, au milieu d'autres micro-mouvements perçus comme machiniques. La conversation entre les deux interlocuteurs communiquant par l'intermédiaire du robot se poursuivait alors sur un mode ludique ("comme si») et devenait d'autant plus riche qu'elle incluait la possibilité pour le robot de rétrograder périodiquement et de revenir à son état de machine, sans que cela soit vécu comme dérangeant. Cette marge de présence faisait partie de la personnalité du Geminoid, qui n'était pas réductible à un simulacre d'humain mais perceptible comme une créature singulière dotée d'une amplitude bien à elle, capable à tout moment de rétrograder à l'état de machine ou de s'upgrader au contraire et être perçue comme le clone d'Ishiguro, voire Ishiguro en personne. Dans ce contexte, un dialogue par l'intermédiaire du Geminoid n'avait rien d'un simple dialogue en face à face entre deux personnes. Le robot dénaturait ou déformait la communication naturelle au point de pousser ceux qui l'utilisaient à s'interroger sur ses fondements. Dès lors que les expérimentateurs avaient accepté sa marge de présence si singulière, de nombreux jeux d'incarnation étaient rendus possibles, qui n'avaient rien à envier aux rituels de possession étudiés d'ordinaire par les anthropologues. Les interlocuteurs pouvaient 
alors jouer à des jeux aussi divers que : "Le Geminoid est mon corps, je suis sa voix» ou encore "Le Geminoid est le corps d'Ishiguro, tu en es la voix", etc. Ces jeux n'auraient pas pu se produire si le robot avait été condamné d'emblée à être assimilé soit à un humain soit à une machine. C'est parce qu'il était ambigu dans sa présence, doté d'une certaine flexibilité à l'usage et que l'on pouvait le reconnaître tantôt comme l'un ou comme l'autre, voire les deux, que le Geminoid était bien plus intéressant pour communiquer qu'un téléphone.

On pourrait croire que le Geminoid confirme l'efficacité de l'anthropomorphisme appliqué qui habite aujourd'hui toute la recherche humanoïde. Mais les mécanismes d'attribution chez l'humain sont d'une telle malléabilité qu'ils autorisent à peu près toutes les identifications, y compris à des machines défaillantes, dès lors qu'on alimente ces mécanismes avec un minimum de stimuli. Et il n'est pas du tout sûr qu'en lui enlevant son visage, en lui mettant des antennes, un troisième œil, trois oreilles, ou en s'inspirant d'une forme totalement étrangère au règne humain ou animal, on n'aurait pas pu avoir avec le Geminoid une interaction tout aussi intéressante. L'intérêt de celui-ci réside peut-être moins au fond dans la simulation du vivant voulue par son concepteur que dans la dévitalisation à laquelle il nous ramène irrésistiblement. En donnant à une machine un visage, Ishiguro a mis en circulation, non pas le double exact d'une personne, mais une image pâle, une esquisse dévitalisée de lui-même censée stimuler les attributions. On a toujours expliqué le goût des Japonais pour les humanoïdes, en dehors des raisons économiques et du vieillissement de la population qu'ils invoquent souvent, par leur propension à l'animisme bien plus que par la volonté de mettre le vivant à distance. C'est un argument aussi banal que répandu chez ceux qui cherchent des clés d'explication "culturalistes». Or les Japonais ne sont pas plus ou moins animistes avec leurs humanoïdes que les hindous avec leurs dieux mécanisés (Grimaud 2008), Hollywood avec ses dinosaures ou le Dr Comandon, ce pionnier du cinéma scientifique français du début du xxe siècle, avec ses films de plantes que l'on voyait grandir en accéléré. Beaucoup d'artefacts robotisés tirent leur vertu du fait qu'ils assèchent le vivant. On veut que les robots en aient les fonctions, mais surtout pas les inconvénients. On verra le problème se poser avec les animaux artificiels comme avec les sex dolls. Leurs utilisateurs, qu'ils soient ou non japonais, veulent toujours plus d'animation, de bruitage, d'interactivité, de sensibilité et de répondant, mais leur intérêt tient au fait que ces objets sont censés offrir les avantages du vivant, sans ses inconvénients. Ils peuvent être la cible de fortes projections anthropomorphiques, mais, en ne prenant en considération que celles-ci, on passe à côté de bien d'autres relations peut-être plus ambiguës mais aussi plus répandues, où l'interactivité et le vivant sont rarement confondus.

Variations sur le principe de dépendance électronique :

à propos d'un robot qui couine quand on le touche

Le fait que les robots s'allument et s'éteignent comme tous les objets électroniques ne facilite pas leur intégration au règne du vivant. Certes, on peut s'accoutumer à leurs vibrations et vouloir qu'ils restent allumés en permanence, mais comment entretenir une relation vivante avec un objet électronique que l'on allume et qui s'éteint sans être accusé de naïveté ? Quand on laisse parler les usages (y compris les siens), on se rend compte à quel point il est aisé de nouer une dépendance à un appareil électronique, de personnaliser et d'aimer son ordinateur par exemple. 


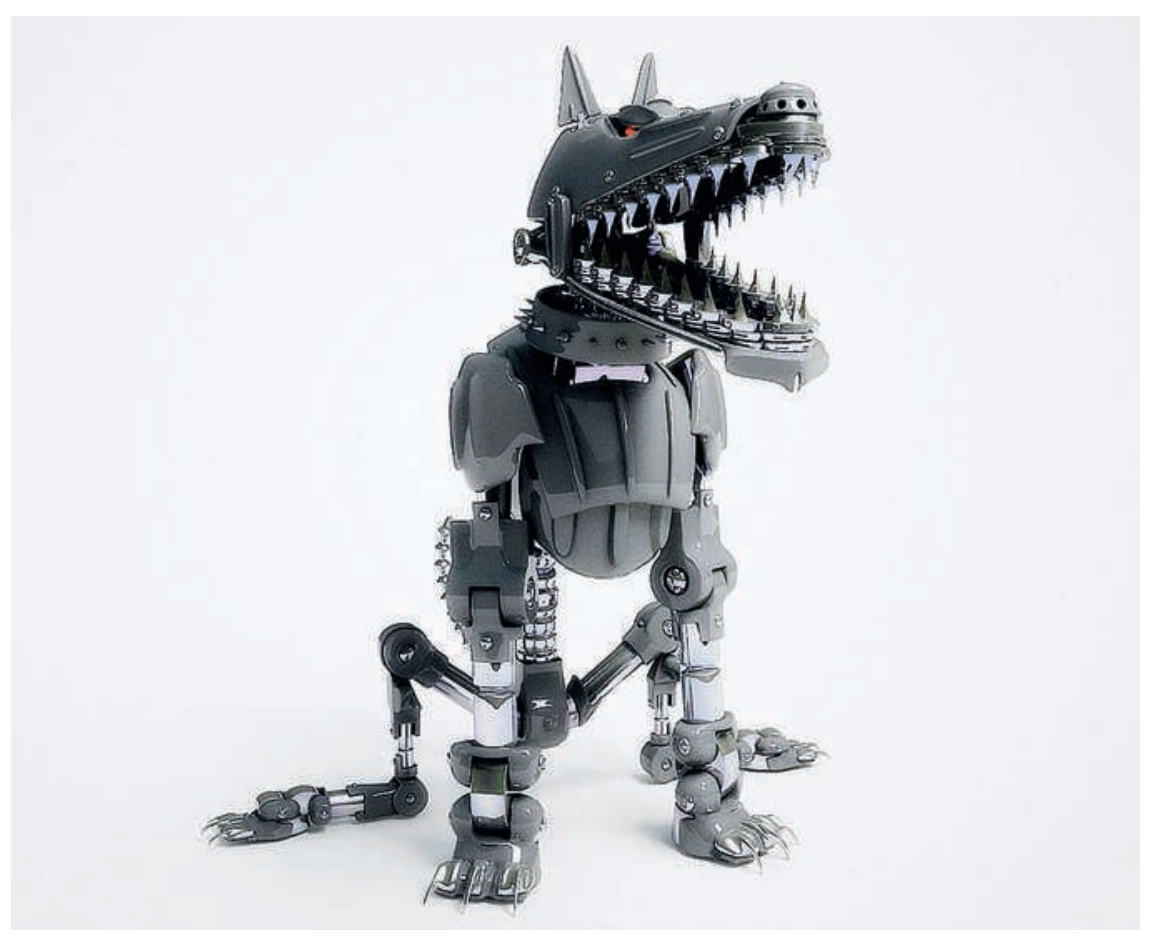

Fig. 7 Dog Robot, Turnado @ TurboSquid.

Mais il n'est pas certain qu'on ait intérêt à considérer comme vivant son ordinateur, son réfrigérateur ou son aspirateur. La problématique de l'attachement englobe le vivant mais ne s'y réduit pas. On peut s'attacher à des objets sans y voir autre chose que des outils et les investir passionnellement ne veut pas forcément dire qu'on les considère comme des êtres. Faisons l'expérience qui consiste, dans cette multitude d'attachements, à glisser de l'un à l'autre et ce sont toutes nos certitudes sur ce que s'attacher veut dire qui tomberont.

Voyons comment on bascule, par exemple, d'un animal de compagnie à un animal artificiel. Paro, le robot phoque, nous aidera à franchir le pas. Dans l'enceinte du musée des sciences de Tokyo, les adolescentes s'arrêtent volontiers plusieurs fois devant son cube en verre et promènent leurs mains sur sa fourrure pour le faire couiner. Il faut avoir caressé une fois dans sa vie ce phoque doté de cinq types de senseurs (tactile, lumière, audition, température et senseur de posture) et qui reproduit les couinements d'un bébé phoque enregistré au Canada par son concepteur japonais. Certes, Paro donne envie qu'on le caresse, mais il n'est pas sûr que nous soyons ici aux antipodes de l'uncanny valley. Zaven Paré et moi sommes à Tokyo dans le hall du constructeur, leader en robotique industrielle. Paro est en démonstration, tout le monde peut le tester. Son pelage blanc et soyeux et sa tétine contrastent avec la froideur mécanique des grues et autres robots industriels qui l'entourent. Zaven s'empare de la peluche. Il faut un peu de temps avant de trouver le mode sur lequel communiquer avec ce robot fait pour vivre en intérieur plus que sur la banquise. Zaven, le visage concentré, les yeux à demi clos, cherche le bon moyen et le trouve très vite. Paro se met à vibrer, couiner, il ouvre et ferme les yeux, et les couinements sont d'autant plus impressionnants que le hall résonne. Zaven ne cache pas son étonnement. "J'ai un chat qui fait un ronronnement exactement pareil. J'étais assez sceptique par rapport 
à ça, mais l'avoir dans les mains, ça change tout. Bon, rapport poids, volume, vibration, il doit avoir le poids d'un bébé d'un mois et demi... " Peu dégourdi, je mets plus de temps à trouver la bonne méthode, la bonne posture. Je demande à Zaven de me guider. «Prends-le comme un enfant!» me conseille-t-il. «Pourquoi pas comme un vrai phoque? " me dis-je. À la réflexion, l'hésitation quant à la manière de le prendre est significative. Paro a une apparence animale. Pourquoi devrait-on le tenir comme un enfant? Il est vrai qu'une tétine en forme de cœur vient parachever son câble d'alimentation. Or c'est lorsqu'on le prend ainsi comme un nouveau-né qu'on est à même de le sentir réagir. Sa forme animale fait qu'on ne s'attend pas à ce qu'il se comporte comme un humain. Ses réactions sont d'ailleurs bien plus imprévisibles. «Prends-le comme ça, me dit Zaven, on se sent plus en sécurité de le porter ainsi!»
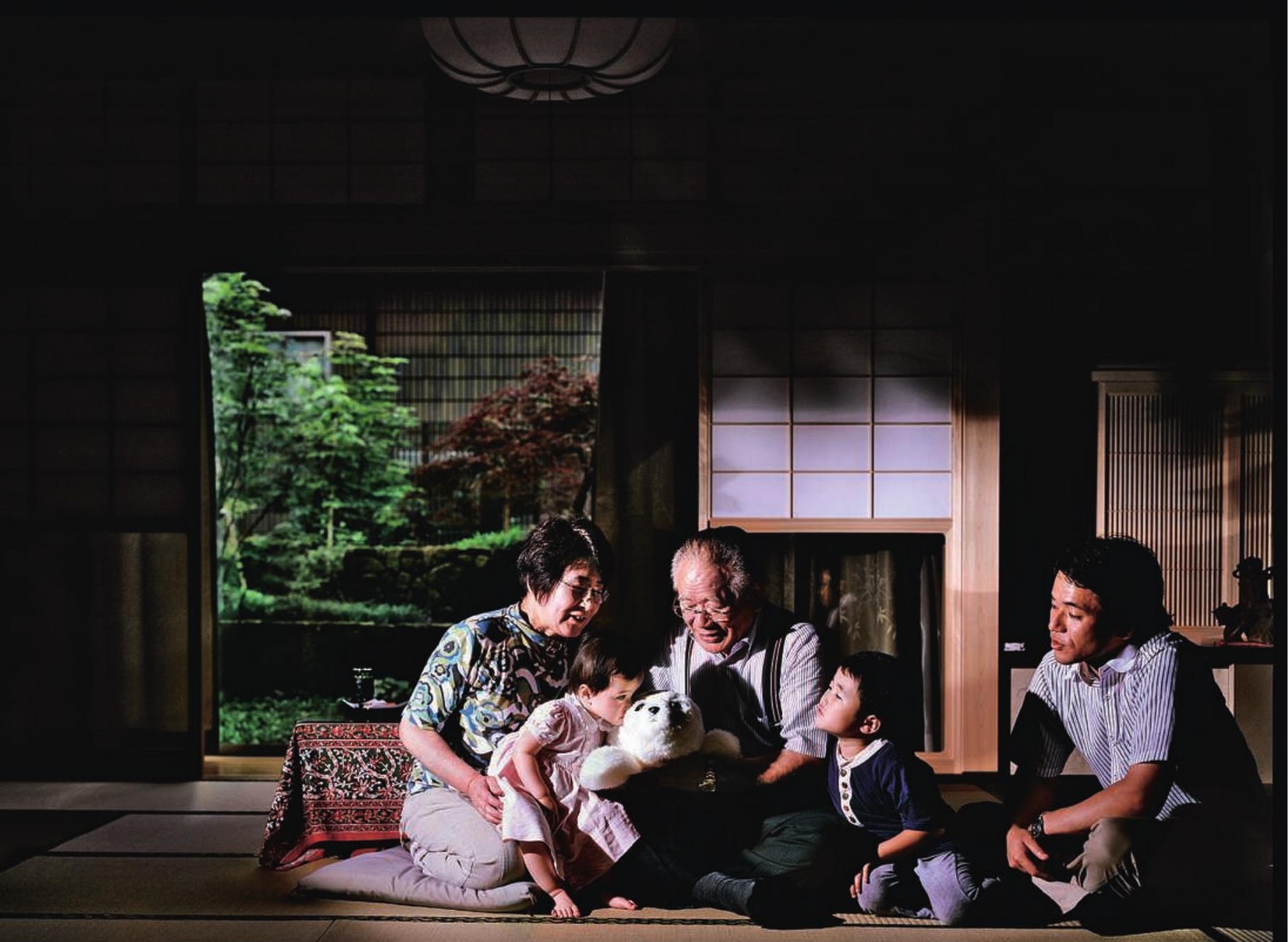

Fig. 8 La famille Shimada autour de Paro @ Max Aguilera-Hellweg. 
Autour du robot en démonstration libre, toute une littérature explique sa finalité et des vidéos très explicites démontrent ses bienfaits pour calmer les fous, atténuer la solitude des vieux, apaiser les femmes enceintes ou accompagner les enfants handicapés. Partout où la présence d'animaux de compagnie pose un problème logistique alors qu'elle est désirée par les patients, Paro peut intervenir et fournir une solution adaptée. On appelle cela de l'animal therapy. Une vidéo nous montre un couple de vieux Japonais dans un bar à sushis, endroit où les animaux ne sont pas autorisés; Paro y est non seulement autorisé, mais bienvenu. Le couple l'a acheté pour rendre sa vie plus joyeuse, nous dit-on. L'homme fait mine de lui tendre sa cuillère quand le phoque couine. Des individus caressent le robot et sont en pleine conversation avec lui dans une maison de retraite. Tout en le caressant, assise devant son téléviseur, une dame dit : "Paro est plus un membre de la famille qu'un robot. Si on pensait à lui comme à un robot, on n'en prendrait pas autant soin. Il peut répondre à ma voix et réagir. Et on peut jouer avec lui et lui apprendre des comportements. Nous n'avons pas d'enfant, nous sommes seuls et Paro grandit, il devient un bon garçon car nous ne le battons jamais. "Vibration, couinement et apprentissage, voilà qui permet l'embrayage dans une relation, un devenir tout autre que la traditionnelle relation de commande : « Je te mets en marche et tu obéis. » Paro donne même l'illusion à ses propriétaires qu'il grandit, de même que d'autres robots peuvent donner l'illusion qu'ils vous regardent. Dans quel type de devenir les propriétaires de Paro s'engagent-ils exactement? "Je te mets en marche et tu évolues", telle est la maxime à laquelle on peut rattacher un nombre croissant de robots. Paro n'est pas le seul capable d'apprendre et il n'est pas le premier. Mais il est ambigu dans la façon dont il entretient les réflexes de la relation de commande la plus traditionnelle. Il reste embourbé dans une relation pavlovienne. Il se situe à mi-chemin entre le chien de compagnie et l'enfant, mais reste tout de même plus proche du premier. "Grâce à ses senseurs tactiles - nous explique son mode d'emploi-, il peut sentir quand on le caresse comme quand on le frappe et, grâce à son senseur de posture, il sent quand on le tient. Paro peut aussi reconnaître la direction de la voix et il est sensible à certains mots comme son nom et les flatteries. Il réagit quand on le félicite grâce à ses senseurs auditifs. Paro peut apprendre à se comporter comme l'utilisateur le préfère. Si vous lui tapotez le dos, Paro s'en souvient et peut reproduire l'action qui a conduit à lui tapoter le dos. Si vous le frappez, Paro s'en souvient et essaie de ne pas reproduire l'action qui l'a conduit à se faire frapper. Dans l'interaction avec les gens, Paro répond comme s'il était vivant, il bouge la tête et les pattes, émet des sons et adopte votre comportement préféré. » Paro cristallise dans son interface plusieurs enjeux de la robotique de compagnie : l'apprentissage, la substitution, la communication sensorielle. Des véritables effets thérapeutiques de Paro, nous ne pouvons pas juger. Considéré par le Livre Guiness des records comme le robot le plus thérapeutique jamais conçu, il est dit qu'il réduit le stress, stimule l'interaction entre les patients et les infirmiers, augmente la relaxation et la motivation. "Je te touche, tu me relaxes. " "Je dis ton nom, tu réponds et je ressens du confort. » Les fabricants d'automates indiens disent la même chose de leurs robots divins aux mouvements lents et hypnotiques, invitant leur public à la sérénité (Grimaud 2008). Autant de variations autour du même mode interactionnel dont la sculpture du Bouddha, selon Mori, était emblématique : "Je te contemple et tu m'apaises.» 
Ce qu'il y a de plus frappant dans la manière dont les utilisateurs racontent leur cohabitation avec Paro, c'est la facilité avec laquelle ils ont depuis longtemps préparé une place à ce robot de substitution, qui n'est ni véritablement assimilable à un enfant qui nous survivra ni à un véritable animal de compagnie. Il faut faire attention ici quand on parle de substitution. Les chiens meurent, tombent malades, il faut les nourrir, les sortir et on ne les autorise pas dans de nombreux lieux publics. Paro ne prétend pas les remplacer. Il est en fait le produit d'une réflexion sur les désavantages des vrais chiens et une réponse au dilemme de notre pauvre condition de mortels. Quand on sent que la mort est proche et que l'on ne veut pas s'embarrasser d'un nouvel animal qui nous survivra, Paro le phoque vient combler le vide. De quel vide s'agit-il exactement? Paro s'est infiltré dans les intérieurs, à côté de l'enfant, du vrai chien et du réfrigérateur. "Je ne vais quand même pas dire bonjour à mon réfrigérateur, mais je dis bonjour à Paro ", dit une vieille dame qui l'a adopté. Il y avait donc la place entre la cuisine et la chambre à coucher pour une créature qui donne envie de la caresser mais qui ne meurt pas, un animal de compagnie sur commande qui n'impose pas les mêmes contraintes d'entretien. C'est le résultat d'une opération d'« hétéromorphose" (plus que d'une anthropomorphose), celle dans laquelle s'est aventurée une grande partie de la robotique contemporaine et qui consiste moins à passer tout ce qui existe à la moulinette de l'échelle humaine qu'à calibrer ses inventions aussi finement que possible pour que celles-ci viennent combler les trous de nos ontologies existantes. Paro n'existait pas, et même si sa place était toute préparée, il fallait l'inventer.

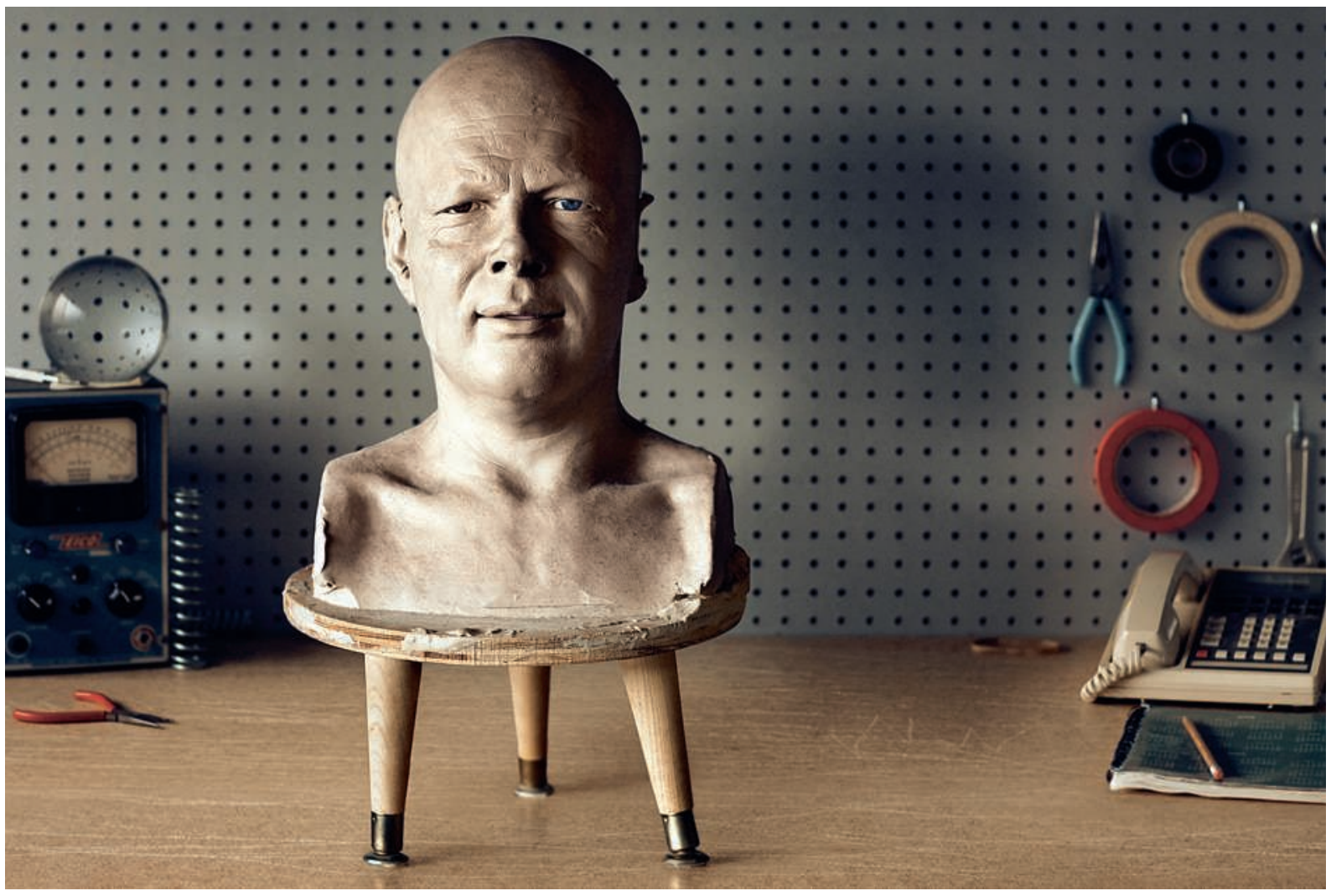

Fig. 9 La tête de Philip K. Dick @ Timothy Archibald. 


\section{« Je te mets en marche et tu obéis 》}

La rhétorique de la substitution (l'idée que les robots sont faits pour se substituer aux êtres humains) est beaucoup moins pertinente pour la création robotique contemporaine que ne l'est celle de l'attachement. Il est vrai que la substitution implique une vision réductrice de la technologie. Elle suppose une certaine forme de dualisme humain-non humain. Or la robotique ne cesse de produire des hybrides, des créatures qui croisent l'ordinateur, le chien et le réfrigérateur, et de repousser toujours plus loin les limites de nos capacités d'attachement. Ensemble, ceux-ci composent une sorte de "para-humanité " dans laquelle on peut progresser par toute une série de glissements, d'analogies, de confusions et d'altérations plus ou moins grandes, du jouet à l'ordinateur, de l'animal de compagnie à l'animal artificiel, du sex toy au mannequin robotisé. Ainsi, Paro s'accommode de la présence d'un vrai chien autant qu'un vrai chien s'accommode de Paro sur un sofa face à un téléviseur. Le chien pourra-t-il dormir encore longtemps sur ses deux oreilles? L'animal artificiel ne l'a pas encore supplanté, il s'est juste ajouté à un environnement déjà saturé d'objets, entre lesquels on passe notre temps à faire des analogies et des transferts de qualité. On pourrait multiplier les exemples montrant que le "para-humanisme» n'est en rien réservé à la communauté des militants pour la protection des ordinateurs ou des robots. Quelle leçon tirer, par exemple, du succès rencontré par un objet célèbre, de quelques années antérieur à Paro, que l'on appelle tamagotchi (du japonais tamago, "œuf " et tchi, qui renvoie à l'affection et à la descendance) ? Souvent assimilé à un animal virtuel, ce gros porte-clés en plastique avec trois boutons et un petit écran à cristaux liquides avait cela de particulier que l'allumer consistait à lui donner naissance et que son arrêt signifiait sa mort. Sur l'écran, une sorte d'œuf palpitant éclosait quand on lui en donnait le signal, avant de devenir une bestiole de 50 pixels que le propriétaire se devait de nourrir, divertir, nettoyer, soigner et même punir.

Dans la plupart des relations qui se développent sur le mode du "comme si", les termes de la relation ne fusionnent jamais complètement. Certes, l'activation de l'objet sur le mode «marche-arrêt» implique ici autrement son utilisateur, tendant vers quelque chose de plus grave, un rapport à la vie et à la mort. Ce n'est pas pour autant que l'usager tombe dans la confusion voulue par les constructeurs. Il suffisait d'avoir oublié plusieurs fois sa créature et réactivé autant de fois le processus de naissance d'un autre œuf pour réaliser que laisser mourir une créature électronique était bien moins grave que laisser mourir un être en chair et en os. On pourrait multiplier les exemples de ce type. Ce n'est pas parce qu'en 2010, les Américains achetèrent 12,5 millions de vibromasseurs, qui vinrent s'ajouter aux 50 millions déjà présents dans les maisons américaines, qu'ils étaient pour autant incapables de faire la différence entre un sex toy et une personne réelle. Il faut distinguer ici entre la confusion ontologique nécessaire « pour la fabrication ", qui joue sur des mécanismes d'attribution et constitue plutôt le problème des fabricants, et ce qu'on pourrait appeler la gradation ontologique, qui concerne davantage l'utilisateur et joue sur d'autres mécanismes auxquels Paro, comme le tamagotchi, nous ont introduits. Les animaux et les jouets pour enfants ne sont pas les seuls concernés. L'industrie du "jouet pour adulte» (sex toy), depuis qu'elle existe, a toujours cherché à tendre vers plus de réalisme et de sensibilité. Dans sa quête de sensations, elle a intérêt à cultiver la confusion autant que les fabricants de Paro alimentent l'amalgame entre les animaux de 
- $\bullet$

4. On pourrait citer pléthore d'exemples de machines organiques conçues par des écrivains, de L'Eेve future d'Auguste Villiers de L'Isle-Adam (1886) aux machines à coudre mangeuses de pilules discutées par Philip K. Dick (1972). compagnie et les animaux artificiels et les concepteurs de tamagotchi militent pour la fusion entre le mode "marche-arrêt» et le mode "vie-mort». Mais lorsqu'on adopte le point de vue de l'usager, on s'aperçoit que l'intégration de tous ces engins a conduit à faire toujours plus de différences. À l'intérieur du monde $\mathrm{du}$ "comme si", tous les objets ne sont pas équivalents, bien au contraire, car ils ne cessent de se différencier tout en se ressemblant. Certes, l'engouement pour Paro comme pour le tamagotchi ou d'autres robots n'est possible que grâce aux capacités d'attribution de leurs usagers et à un certain degré de confusion initial, mais quand on y regarde bien, leur survie n'est envisageable que parce que leurs usagers les intègrent dans des échelles de plus en plus graduées de traitement des objets, des êtres, des machines et de leurs hybrides.

Si on comprend l'intérêt que les fabricants ont à cultiver la confusion plutôt que la gradation, le but est plus rarement au fond d'opérer une véritable substitution que l'ajout ou l'intégration dans une panoplie d'objets déjà existante. Un animal artificiel ne pourra s'intégrer dans un appartement que s'il se modèle suffisamment sur un véritable animal, de la même façon que l'industrie du jouet érotique a plus de chance de s'attirer des clients en se calibrant sur un univers de sensations déjà connues plutôt qu'en assumant explicitement ses effets d'altération de la relation amoureuse. En réalité, l'altération est aussi motrice que la confusion, mais elle n'est pas toujours aussi vendeuse. Examinons de plus près ce qui se passe dans l'univers des "jouets pour adultes". Ce dernier est bien plus qu'un produit dérivé de la robotique humanoïde. Il en est devenu dans les dernières décennies une branche à part entière. Il n'est pas rare de voir au Japon des stocks entiers de sex toys dans les entrepôts des laboratoires de robotique. Le roboticien à l'origine du Geminoid était lui-même convaincu que l'érotisme était un domaine d'avenir. Et il n'était pas le seul.

En 1968, David Levy, maître international en jeu d'échecs, lança un défi à quatre géants de l'intelligence artificielle afin de stimuler la conception de logiciels de jeux d'échecs. On connaît la suite de l'histoire : la célèbre défaite de Garry Kasparov contre Deep Blue en 1997. Ce que l'on sait moins, c'est que Levy est passé dix années plus tard à un tout autre type de prophétie. Dans Love and Sex with robots. The evolution of human-robot relationships (2007), qui n'est autre que sa thèse de doctorat en intelligence artificielle, il prédit l'explosion des robots érotiques et déclare qu'il sera bientôt possible, et même courant, d'entretenir avec des robots de fortes relations de dépendance affective. Cette thèse s'appuyait sur une connaissance apparemment approfondie du champ et confirmait l'intuition de beaucoup de roboticiens travaillant sur des humanoïdes. L'industrie des jouets érotiques constituait déjà l'un de leurs principaux terrains de jeu. Mais l'aspect le plus intéressant du livre de Levy était moins sa prophétie que la démarche qu'il employait pour nous convaincre de sa réalisation imminente. Au terme d'un cheminement explorant une large panoplie de robots existants, tout aussi uncanny les uns que les autres, l'érotisation des humanoïdes paraissait être une innovation tellement minime qu'elle ne faisait plus problème sur un plan ontologique. Tout le monde semblait y avoir été plus ou moins préparé par plusieurs siècles de littérature", et l'avoir déjà inventée avant qu'elle ne se réalise concrètement.

Beaucoup de problèmes qui concernent la robotique en général dans la manière dont elle doit envisager le devenir social de ses objets se retrouvent cristallisés "en miniature » dans les machines érotiques, mais aussi en grandeur nature. On y retrouve la même tension entre attraction et répulsion, la même tendance à jouer $\mathrm{du}$ fait que ce qui nous repousse peut aussi nous attirer, la même dépendance de 

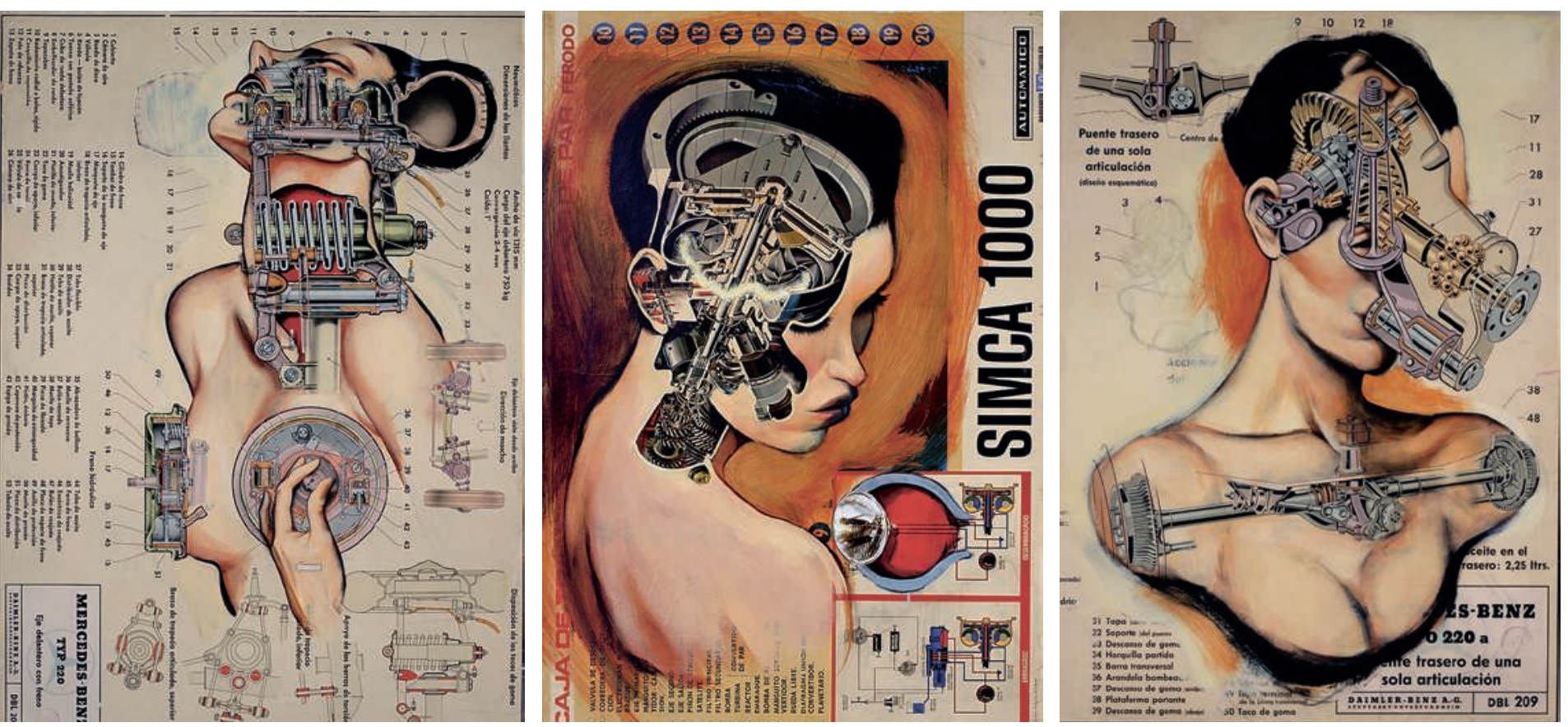

Fig. 10 à gauche : Anatomías - Fernando Vicente - Sintonizando; au centre : Anatomías - Fernando Vicente - Máquina sueños; à droite: Anatomías - Fernando Vicente - Medidor de los sentidos Fernando Vicente - www.fernandovicente.es

l'artefact par rapport aux mécanismes d'attribution de leurs usagers et la même prétention de l'artefact robotique à faire « table rase » autour de lui dans le contexte où il vient s'infiltrer. Pourquoi a-t-on l'impression de flirter ainsi avec une limite, une frontière, dès lors que l'on convoque la robotique au cœur de l'intime? C'est qu'il est difficile d'être purement matérialiste en matière d'amour et de sexualité. Le sex toy, pourtant, ne semble toucher à première vue à aucune limite, les pénis et les vagins artificiels confortent le corps humain comme référence ultime. Les designers éprouvent ici les mêmes difficultés que dans la robotique en général à concevoir des corps entiers ou à penser l'espace de la relation sexuelle. Ils ne peuvent faire guère plus que de s'y infiltrer et font preuve de bien plus d'efficacité en décomposant le corps en fragments, en pensant d'abord et avant tout organes et matériaux, opérations et actions, modalités de contrôle et de commande, variations d'intensité, nombre de coups par minute. La pénétration, l'aspiration, la vibration et la circonvolution constituent ici les modes privilégiés de l'attachement. Il existe aujourd'hui quasiment autant de machines que d'applications, qui viennent principalement du Japon et des États-Unis, de fabricants ayant pignon sur rue mais aussi d'amateurs qui fabriquent des machines dans leur garage, détournent des appareils électroménagers et des aspirateurs ${ }^{5}$ (Archibald 2005).

Contrairement à ce qu'on pourrait croire, les bricoleurs américains qu'Archibald a photographiés avec leurs machines ne sont pas des pervers polymorphes, mais des Américains moyens, souvent mariés, qui ont commencé par concevoir des machines durant leur temps libre pour leur femme, comme un hobby, avant de les distribuer ensuite auprès de leurs amis. Certes, on peut se demander pourquoi vouloir compliquer autant l'acte sexuel avec des appareils. Tandis que ceux qui n'y recourent pas perçoivent l'intrusion de l'outil comme une contamination ou une pollution du rapport amoureux, leurs adeptes, au contraire, invoquent

\footnotetext{
-

5. David Levy fournit un bon état de l'art sur le sujet (2007), tout comme le blog d'Agnès Giard. La journaliste a rendu compte pendant plusieurs années sur le site du journal Libération, avec une grande finesse d'écriture et d'analyse, de nombreuses innovations sur le marché du jouet érotique et qui touchent de près ou de loin à la robotique.
} 


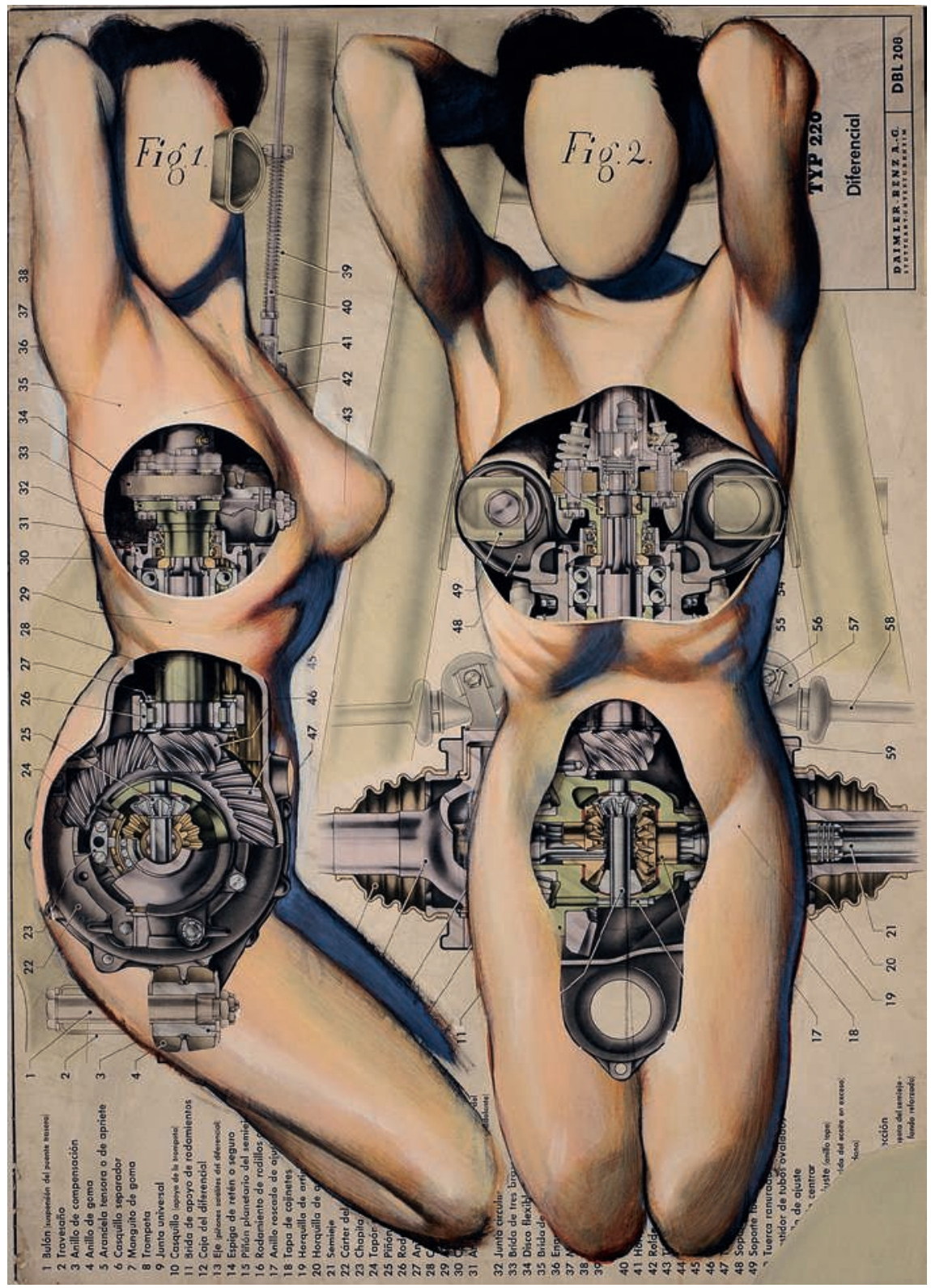

Fig. 11 Anatomías - Fernando Vicente - fig 1 et 2. Fernando Vicente - www.fernandovicente.es.

le fait qu'avec une telle philosophie, l'ordinateur n'aurait jamais vu le jour. On retrouve donc dans ce domaine tous les débats qui agitent l'univers des technologies en général; on y décèle aussi l'ambiguïté des usages, puisqu'on peut imaginer recourir à des sex machines pour de multiples raisons, pour remédier à un manque, parce qu'elles offrent un substitut commode, par simple curiosité ou parce qu'elles permettent d'explorer un continent de sensations insoupçonné. On tentera de répondre ici à deux questions seulement. La première, c'est pourquoi et comment passe-t-on du sex toy au mannequin robotisé? Comment qualifier ce glissement par rapport à ceux que l'on a déjà examinés? Et la seconde : en quoi le recours à des machines est-il susceptible de modifier les rapports de pouvoir dans la relation charnelle? 
Rappelons-nous l'exemple de Paro. Un animal artificiel crée une nouvelle forme d'attachement "animal compatible ", moins contraignante et plus lâche, permettant d'éviter de faire face à la mort de l'animal. C'est justement parce qu'il n'est pas un animal réel et qu'il n'en présente pas les inconvénients qu'on l'a adopté aussi facilement. Les honey dolls japonaises, quant à elles, doivent leur succès moins au fait qu'elles sont des substituts (elles le sont sans aucun doute), qu'au fait qu'elles ne sont pas des personnes et qu'elles donc sont bien plus soumises et contrôlables. Comme le raconte Agnès Giard dans l'une de ses chroniques, les honey dolls sont capables d'effectuer une fellation à intensité variable, elles sont équipées d'un mécanisme de rotation au niveau des hanches afin de s'adapter à un maximum de positions, on peut aussi les faire gémir en leur pinçant les seins; elles sont par ailleurs dotées de senseurs et d'un module vocal avec quatre voix différentes; on peut enregistrer soi-même la voix d'une femme, afin de les personnaliser; les têtes sont interchangeables et les dents sont souples afin d'éviter tout accident. Certes, leurs capacités de réaction donnent une vision pour le moment bien misérable du rapport sexuel. Mais c'est que les honey dolls reproduisent un rapport de pouvoir aussi vieux que l'histoire de l'humanité. S'il nous faut un critère pour juger de l'inédit en robotique, c'est bien celui-ci : dans quelle mesure l'interface reproduit-elle des rapports de commande existants ou les modifie-t-elle? On le verra par la suite, les sex toys ont une capacité d'érosion sur les rapports de contrôle bien plus importante que les mannequins robotisés. La nouveauté des poupées est ailleurs. Elles ne sourient pas et gardent la bouche semi-ouverte. Comme l'explique Agnès Giard, elles ressemblent à des fantômes, ce qui, dans l'esprit des fabricants japonais, doit permettre d'y projeter ses fantasmes et de s'identifier. Il faut juste laisser s'épanouir les attributions, ici comme ailleurs. C'est dans cet espace de présence faible, celui où s'épanouissent les fantômes, que les honey dolls s'enracinent et viennent accrocher leurs utilisateurs.

On pourrait penser que tous les éléments sont là depuis bien longtemps, qu'il suffisait de les assembler ${ }^{6}$, mais le glissement vers la reproduction d'un corps humain dans son entier ne va pas de soi. Le saut mental qu'il faut opérer pour passer du jouet érotique au mannequin robotisé n'est peut-être pas tout à fait comparable à celui qu'il a fallu franchir pour passer de la machine à écrire à l'ordinateur. Il faut reconnaître que lorsque les techniques se répondent ainsi, qu'elles se stratifient en s'appuyant sur celles qui leur ont déjà préparé le terrain, ce sont d'infimes glissements que nous discutons. Mais dans les cinétiques de l'attachement, chaque détail, chaque micro-mouvement et changement de sensation compte. Et certains basculements, en apparence minimes, constituent des fossés ontologiques. Si l'on adopte pour critère de jugement des innovations robotiques leur capacité à déplacer, troubler ou réformer les rapports de commande existants, il faut reconnaître que fabriquer des poupées soumises, aussi interactives soient-elles, est beaucoup moins inédit que de développer des robots industriels qui nous poussent à réviser notre conception du travail et ses modalités d'organisation. De plus, il n'y a pas de raison de penser que l'avenir du mariage entre la robotique et la sexualité n'est pas au fond, comme dans l'industrie automobile ou dans le mobilier, dans plus de délégation aux utilisateurs des moyens de composer leur propre véhicule ou leur propre cuisine. C'est ainsi en tout cas que beaucoup de designers français mettent à distance l'entêtement des Japonais à fabriquer des humanoïdes. Les sex toys appartiennent à l'histoire de l'outillage et offrent, à ce titre, bien plus de manœuvrabilité et de possibilités d'usage en tant

\section{(1)}

6. Dans La Vie sexuelle de notre temps (1906), Iwan Bloch, le père fondateur de la sexologie, raconte: $\ll$ On pourrait citer de nombreux exemples d'actes de fornication réalisés par des imitations artificielles du corps humain ou de fragments corporels. II existe de véritables Vaucansons en matière de technologie pornographique, des mécaniciens ingénieux qui conçoivent, à partir du caoutchouc et d'autres matériaux plastiques, des organes mâles et femelles au service de la fornication et que l'on appelle des hommes et des femmes du voyage. Les plus spéciaux sont les organes génitaux qui imitent la nature avec une grande précision. Même la sécrétion des glandes de Bartholin est imitée, grâce à un tube pneumatique alimenté par de I'huile. De la même façon, grâce à des appareils fluides, l'éjaculation de la semence peut être reproduite. De tels êtres humains artificiels sont en vente dans les catalogues de fabricants d'articles en caoutchouc parisiens. » 
- -

7. Dave Lampert, professeur de danse et inventeur d'une célèbre machine appelée Sybian Machine (1985), qui se vendit très bien dans les communautés d'échangistes chrétiens aux États Unis, justifia ainsi son invention : « J'ai acheté beaucoup de livres sur le plaisir féminin et la plupart de ces livres répétaient la même bêtise, la même gigantesque et aberrante méprise, que l'on voit dans les films $X$ et que la plupart des hommes tiennent pour une vérité [...]. L'humanité ne s'en sortira jamais tant que ce mythe perdurera [...]. Ma machine est agitée de mouvements sur sa base. La majorité des sex machines ne font que coulisser d'avant en arrière : elles fonctionnent sur le principe de la pénétration. Mais ce n'est pas le va-et-vient qui compte pour une femme, c'est l'intensité des vibrations... » que membres portatifs qu'assemblés dans un mannequin robotisé reproduisant une relation de soumission et qui ne sera rien d'autre qu'un assemblage d'éléments (une bouche, un pénis, un vagin, des seins, etc.) idéalement "personnalisables ". De même qu'on constate que penser le corps en fragments est à la fois plus innovant et plus acceptable, il faut reconnaître le rôle joué ici par la tension entre anthropomorphisme et zoomorphisme. Ce dernier est en fait aussi moteur dans la robotique humanoïde à orientation érotique qu'il l'est dans la robotique de compagnie. On pourrait citer de nombreux exemples pour illustrer cette idée. Christophe Luxereau, un artiste français, imagina en 2007 un sex toy interactif au design ergonomique de calamar, capable d'enfoncer ses pédoncules munis de petites ventouses dans tous les orifices. Il n'était pas le premier et surtout pas le dernier à fantasmer sur des formes de fusion animale et à explorer d'autres esthétiques. On ne compte plus aujourd'hui les vibromasseurs de toutes les tailles, en forme de pénis, de doigt ou d'œuf, dont certains se commandent à distance, et les sex toys en forme de bouche, munis de ventouses et de mécanismes aspirants. Une simple visite au musée de l'Érotisme, à Paris, suffit pour s'en rendre compte. Beaucoup de ces objets ont des formes explicitement animales et jouent sur un mélange de peur et de fascination. D'autres engins, qui reproduisent des fausses mains, des tentacules, des langues qui salivent ou des gorges aspirantes avec des tubes gélatineux et visqueux, promettent des massages pour les plus doux et des circonvolutions infernales, des tournoiements de robot-mixeur, des mouvements de trayeuse ou d'essoreuse pour les plus extrêmes.

Il suffit d'un rien pour que l'expérience de substitution bascule dans l'étrangeté, puis de l'étrangeté dans la cruauté7. Certes, les vertus des machines érotiques sont d'abord physiques (elles sont réputées infatigables, insatiables, ajustables à l'infini et promettent des effets inconnus), mais leur succès repose comme pour d'autres artefacts robotiques sur une forme de contact «électro-métaphysique ». Par secousses et vibrations interposées, elles font vaciller les fondements de l'amour humain et animal. Elles le retravaillent comme jamais dans l'histoire des techniques, poussant les hommes à s'interroger de manière réflexive sur ce qu'est l'amour, sa consistance, et sur les modalités sensorimotrices de ce qui nous attache les uns aux autres. Les frontières de l'amour vacillent, on ne sait plus vraiment ce que c'est, s'il est ici, là ou ailleurs. De la même façon et plus largement, on ne s'est jamais autant posé la question de savoir ce qu'être humain signifie que depuis qu'on fabrique des humanoïdes.

\section{Conclusion}

Les robots obligent toujours à un moment ou un autre, soit au cours de leur conception soit dans leur usage, à repenser la pratique ou l'environnement dans lequel ils viennent s'inscrire. Il ne faut pas croire que la question de l'attachement ne concerne qu'une petite partie de la robotique, celle qui se situe explicitement sur le terrain des passions. Prenons l'autre extrémité du spectre, les robots apparemment les plus dénués d'enjeux affectifs, car attachement et détachement vont de pair. Les engins militaires dessinés par Norbert Wiener, l'un des pères fondateurs de la cybernétique, et qui reposaient sur des boucles de rétroaction, marquèrent une étape décisive dans la recherche du meilleur moyen d'accomplir sa mission avec le plus complet détachement (Wiener 1948). Si la robotique constitua l'un des investissements majeurs de l'armée américaine au début du xxi siècle, c'est 


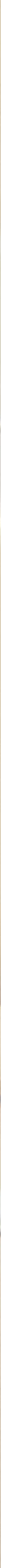

Fig. 12 Nirvana avec The Thrillhammer, à la maison close Chicken Ranch Brothel, Parhump, Nevada @ Timothy Archibald. 
que les robots militaires présentaient un grand nombre d'avantages par rapport à des soldats en chair et en os. Ce n'est pas pour rien que la cybernétique de Wiener s'est d'abord épanouie dans le champ militaire. C'est ici que l'épuration de l'action envisagée comme opération y est la plus forte. L'un des textes les plus populaires de l'hindouisme, la Bhagavad-Gîtâ, met en scène le guerrier Arjuna en plein doute sur le sens de la guerre qu'il conduit et le dieu Krishna lui expliquant la méthode yogique pour accomplir son acte avec détachement. Plusieurs centaines de pages de méditation philosophique sont nécessaires pour que le guerrier se mette dans les conditions voulues. Les engins robotisés n'ont pas besoin d'un tel conditionnement.

Heinrich von Kleist avait remarquablement formulé, dans son célèbre texte sur le théâtre de marionnettes (1801), tout le potentiel que l'on peut tirer d'un mécanisme pur, dénué de sensibilité émotive. Une marionnette pourrait bien être supérieure à l'être humain d'un point de vue cinétique, puisqu'elle peut reproduire un geste à la perfection bien mieux qu'un danseur en chair et en os luttant contre l'anxiété et toutes les choses mentales qui lui polluent l'esprit au moment où il entre en scène, et qui viennent entraver la pureté de son mouvement. Wiener était kleistien sans le savoir, les armées du xxi ${ }^{\mathrm{e}}$ siècle le sont aussi lorsqu'elles s'appuient sur les machines pour dépassionner les actions de guerre. De réflexivité, les robots sont dénués. Quand ils sont bien conçus, ils font ce qu'on leur dit. Tel est l'argument de leurs promoteurs. Les robots militaires offriraient du détachement "yogique " sur commande sans avoir besoin de longues heures de méditation, permettant de se préserver instantanément du contact avec la cible. Mais, au vu de ce qui précède, on comprendra que la quête froide du détachement et la réduction de l'action à une pure opération ne sont pas le seul apanage des militaires.

Il n'est pas du tout certain en effet que les liens noués avec des animaux de compagnie robotisés tout comme avec des robots érotiques ne soient pas le lieu d'une tension similaire entre attachement et détachement. Pendant que la robotique humanoïde fait tout pour doter ses créatures de capacités sensorielles, les amateurs d'animaux robotisés comme de robots érotiques ne s'en emparent pas toujours pour les confondre avec de l'existant, mais aussi pour «dépassionner " certains objets ou "désincarner » certains liens et graduer toujours plus les relations qui les entourent. Cette flexibilité d'usage qui fait qu'un robot peut être tantôt relégué au statut de dispositif de commande désincarné, tantôt assimilé à de l'humain sur le mode du "comme si », constitue toute l'ambiguité des artefacts robotiques dès lors qu'on prend la peine de les resituer au sein des cinétiques de l'attachement qui leur donnent sens.

Le débat a changé depuis le fameux test de Turing (1950), où seulement deux options s'offraient aux machines : devenir humaines au prix d'un tour de prestidigitation ou rester condamnées à leur état de machine. En dehors de la confusion ontologique (entre l'humain et la machine), toujours de courte durée, on a vu qu'une rencontre réussie avec un humanoïde nécessitait d'autres éléments. La robotique travaille la perception du micro-mouvement, la vibration, le répondant, ainsi que le désir du contact. Entre absence et présence, apparition et disparition, animé et inanimé, veille, sommeil et rupture du contact, chaque robot esquisse un rapport et engage l'interactant à sa manière dans l'exploration de nouvelles gammes de relations, ni simplement assimilables à du vivant, ni purement objectales ou machiniques. La plupart des humanoïdes sont des créatures «sollicitudinaires», comme disait Étienne Souriau (1956), c'est-à-dire qui 
n'existent que par et dans leur dépendance. Mais le mode "marche-arrêt » n'est qu'un mode parmi d'autres de déclenchement de la sollicitude.

C'est que la relation de commande, à la base de beaucoup d'artefacts robotiques et qui en constitue le noyau originel, est d'une exceptionnelle plasticité. Passé le mythe de l'autonomie absolue, la robotique invite à explorer une gamme d'autonomies relatives et de modes de contrôle dont on peut se demander s'ils sont toujours réductibles à la maxime : "Je te mets en marche et tu obéis. " La robotique ne débouche pas seulement sur une théorie graduée des attachements, elle contient en elle toute une anthropologie du contrôle qui reste largement inexplorée. La maxime « Je te mets en marche et tu obéis » a ceci de remarquable qu'elle ne concerne pas seulement tout objet se connectant par un interrupteur, mais qu'elle se fond très bien dans nombre d'autres relations, qu'il s'agisse d'une relation ludique avec un animal, de l'amour avec son partenaire, de la relation de l'artiste à son outil, etc. La problématique de la commande est partout et on ne la soupçonne pas forcément derrière l'empathie, l'apitoiement, la coopération, l'agression ou encore la séduction. Or la robotique se développe quasiment exclusivement en pensant «commande». Et lorsqu'elle se situe sur le terrain de l'attachement, elle pense à la fois commande et émotion ou affect, commande et empathie, commande et coopération, commande et séduction, etc. Elle décortique les rapports de contrôle et les besoins humains en relations programmables qu'elle matérialise dans des interfaces de câbles et de circuits. Comment le rapport basique «Je te mets en marche et tu obéis " peut-il se transformer en autre chose? Comment la commande vient-elle se loger dans l'empathie, dans l'amour ou dans la séduction et comment la faire évoluer? C'est sans aucun doute l'une des questions majeures auxquelles la robotique est confrontée lorsqu'elle se situe sur le terrain de l'attachement. Et si la robotique humanoïde, à force de faire de l'anthropomorphisme appliqué, avait en réalité généré tout sauf un anthropomorphisme généralisé? Constatant la puissance du cinéma à révéler les mouvements subtils des choses qui nous entourent, Jean Epstein écrivait : "Un animisme étonnant est rené au monde. Nous savons désormais pour le voir que nous sommes entourés d'existences inhumaines. " (1974 [1935] : 251) On pourrait sans doute dire la même chose de la robotique. Peuplant notre entourage de créatures hybrides proches mais jamais complètement équivalentes, celle-ci a œuvré sans le savoir à un décloisonnement, une perturbation et une diversification de notre rapport au vivant. Distribuant de la vibration électrique partout où on peut en loger, elle a créé les conditions d'émergence de nouveaux attachements, explicitement technologiques, exceptionnellement flexibles et accidentels, fondés sur la possibilité d'un contact immédiat, mais hanté à tout moment par le risque de le perdre.

LESC-CNRS-ARTMAP

emmanuel.grimaud@gmail.com

mots clés / keywords : attachement // attachment - interaction homme-robot // human-robot interaction - anthropomorphisme // anthropomorphism • animisme technologique // technological animism. 


\section{Bibliographie}

Anderson, Alan Ross (dir.)

1983 Pensée et Machine. Paris, Champ Vallon.

Archibald, Timothy

2005 Sex Machines. Los Angeles, Process.

Bloch, Iwan

1906 La Vie sexuelle de notre temps.

Berlin, Marcus Verlagsbuchhandlung.

BRAITENBERG, Valentino

1984 Vehicles: experiments in synthetic psychology. Cambridge, MIT Press.

\section{Chklovski, Viktor}

2008 L'Art comme procédé. Paris, Allia (édition originale 1917).

Dick, Philip K.

1998 « Androïde contre humain », in Si ce monde vous déplaît... et autres écrits. Paris, L'Éclat (édition originale 1972).

Epstein, Jean

1974 « Photogénie de l'impondérable » (1935), in Écrits sur le cinéma, t. I.

Paris, Seghers.

Grimaud, Emmanuel

2008 Dieux et robots. Apt, L'Archange

Minotaure.

2009 « L'animisme technologique : de la vie et mort des machines en général, de celles de Zaven Paré en particulier », in Cyber Art. Rio de Janeiro, Stamppa.

Grimaud, Emmanuel et PARÉ, Zaven 2011 Le jour où les robots mangeront des pommes. Conversations avec un androïde, Paris, Petra.

Gunderson, Keith

1964 « The Imitation Game », Mind 73 : 234-245.
Heider, Fritz et Simmel, Marianne

1944 « An experimental study of apparent behavior », American Journal of Psychology $57: 243-259$

\section{KLEIST, Heinrich von}

1998 Sur le théâtre de marionnettes. Paris, Mille et Une Nuits (édition originale 1801).

\section{LAFITTE, Jacques}

1972 Réflexions sur la science des machines.

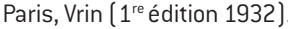

\section{Levy, David}

2007 Love and Sex with robots. The evolution of human-robots relationships. New York, Harper et Collins.

LiBin, Alexander et Elena

2004 « Robotic Psychology », in Encyclopedia of applied psychology, vol. III. Oxford, Elsevier : 295-298.

\section{Mannoni, Octave}

1969 « Je sais bien, mais quand même... » (1963), in Clefs pour l'imaginaire.

Paris, Seuil [《Points 》].

MoRI, Masahiro

1970 « Bukimi no tani. The uncanny valley », Energy ? (4) : 33-35.

2005 « On the uncanny valley », Proceedings of the Humanoids-2005 workshop. Views of the Uncanny Valley, Tsukuba.

PARÉ, Zaven

2011 «Saya ou l'impossible virtuosité », Ateliers d'anthropologie 35, http://ateliers.revues.org/887?.

\section{Peirce, Charles Sanders}

1878 «Comment se fixe la croyance ? 》, Revue philosophique (édition originale 187?).
ROBERTSON, Étienne-Gaspard 2000 Mémoires récréatifs, scientifiques et anecdotiques d'un physicien-aéronaute, t. I : La Fantasmagorie. Paris, Café Clima [édition originale 1831].

\section{SCHAFFER, Simon}

1999 «Enlightened Automata », in William Clark, Jan Golinski et Simon Schaffer (éd.), The Sciences in Enlightened Europe. Cambridge, Cambridge University Press.

\section{SOURIAU, Étienne}

2009 « Le mode d'existence de l'œuvre à faire », in Les Différents Modes d'existence.

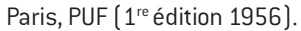

\section{Suler, John}

1996 « Mom, Dad, computer. Transference reactions to computers », in The Psychology of cyberspace, http://users.rider.edu/ffsuler/ psycyber/comptransf.html.

Turing, Alan

1950 « Computing machinery and intelligence », Mind 36 : 433-460.

TuRkle, Sherry

2005 The Second Self. Computers and the human spirit. Boston, MIT Press

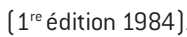

Villiers de L'Isle-Adam, Auguste 1972 L'Ève future. Genève, Éditions de Crémille (édition originale 1886).

WiEner, Norbert

1948 Cybernetics. Or control and communication in the animal and the machine. Cambridge, MIT Press.

\section{ZifF, Paul}

1959 «Les sentiments des robots 》, Analysis 19(3) ; rééd. in Anderson 1983. 


\section{Résumé / Abstract}

Emmanuel Grimaud, Androïde cherche humain pour contact électrique. Les cinétiques de l'attachement en robotique Toute interaction avec un robot gagne à être étudiée à l'échelle où l'observe Masahiro Mori, c'est-à-dire comme une cinétique de l'attachement qui peut être suivie en temps réel dans ses fluctuations d'intensité. Mais une interaction avec un robot anthropomorphe n'est-elle réussie qu'à la condition que nous oubliions, à un moment ou à un autre du processus, qu'il s'agit d'une machine? La question se pose de manière accrue à la robotique lorsque celle-ci se situe sur le terrain des passions. On prendra ici plusieurs exemples (humanoïdes, animaux artificiels, machines érotiques], qui montrent que des liens d'attachement, des jeux communicationnels et parfois même des relations passionnelles peuvent se développer avec des robots, sans qu'il soit nécessaire de les ranger à tout prix et définitivement dans la catégorie « humains» ou «machines». Et si les échelles de traitement des objets, des êtres, des machines et de leurs hybrides peuvent être très variées selon le contexte où l'on se situe, elles sont souvent bien plus souples, graduées et prêtes à s'ouvrir à des relations troublantes que la vieille opposition de l'humain et de la machine ne le laisserait penser. Aussi la robotique nous invite-t-elle à repenser ce que s'attacher veut dire, avant tout débat sur l'apparence même de ses créatures.
Emmanuel Grimaud, Android in search of a human for electrical contact. The kinetics of binding in robotics - Every interaction with a robot calls for a study in terms expressed by Mori, that is, as a kinetic of binding that sudden ontological reversals affect in real time. The same succession of observations informs it consistently, starting with a stage approach and a moment of deep confusion between man and the machine ("this may well be human"), followed by a disassociation phase ("this is just a machine"). The interaction succeeds if it prompts a variety of communicational games where the robot is never classified once and for all in the category of "human" or "machine" in order for a relationship to be established. In this way, interaction with a robot amounts to entering incidentally a small metaphysical theatre characterized by a certain ontological flexibility. Viewed through this perspective, the humanoids examined in this text and their byproducts conceived to generate attachment (pets, erotic robots] are never creatures like any other. 